\title{
THE TOP TEN VALUES OF HARMONIC INDEX IN CHEMICAL TREES
}

\author{
Aliye Zolfi ${ }^{1}$, Ali Reza Ashrafi ${ }^{1}$ and Sirous Moradi ${ }^{2}$ \\ ${ }^{1}$ Department of Mathematics, Faculty of Mathematical Sciences, \\ University of Kashan, Kashan 87317 - 51167, I. R. Iran \\ ${ }^{2}$ Department of Mathematics, Arak University, Arak, I. R. Iran \\ E-mails: ashrafi_1385@yahoo.co.in, ashrafi@kashanu.ac.ir
}

(Received July 3, 2014)

\begin{abstract}
Let $G$ be an $n$-vertex graph with degree sequence $d_{1}, d_{2}, \ldots, d_{n}$. The harmonic index $H(G)$ is defined as $n / I(G)$, where $I(G)=\sum_{i=1}^{n}\left(I / d_{i}\right)$. In this paper the top ten values of harmonic index in the set of all chemical trees of order $n$ are determined.
\end{abstract}

Keywords: Harmonic index, chemical tree.

\section{INTRODUCTION}

We use West [1] for terminology and notation not defined here and consider finite simple connected graphs only. Suppose $G$ is such a graph with $V(G)=\left\{v_{1}, v_{2}, \ldots, v_{n}\right\}$. If we sort vertices of $G$ in such a way that $\operatorname{deg}\left(v_{1}\right) \leq \operatorname{deg}\left(v_{2}\right) \leq \ldots \leq \operatorname{deg}\left(v_{n}\right)$ then the sequence $\left(d_{1}, d_{2}\right.$, $\left.\ldots, d_{n}\right)$ is called a degree sequence for $G$, where $d_{i}=\operatorname{deg}\left(v_{i}\right), 1 \leq i \leq n$.

A graph invariant is any function on a graph that does not depend on a labeling of its vertices. A big number of different invariants have been employed to date in chemistry for solving some chemical problems. Here we are interested to the harmonic index defined as $H(B)=n / I(B)$, where $I(B)=\sum_{i=1}^{n}\left(1 / d_{i}\right)$, for a graph $B$. This topological index was introduced by Narumi [2].

A chemical tree is a tree in which every vertex has degree at most 4 . We denote by $g(n)$, the set of all $n$-vertex chemical trees. It is easy to see that if $A$ and $B$ are two elements of $g(n)$ with the same degree sequence then $H(A)=H(B)$. This is motivation for defining an equivalence relation $\sim$ on $g(n)$ by $A \sim B$ if and only if $A$ and $B$ have the same degree sequence. Suppose $\mathcal{C}(\mathrm{n})$ denotes the set of all equivalence classes of $\sim$ on $\mathcal{C}(n)$ and $T_{1}, T_{2}$ $\in \mathcal{C}(n)$. Define $T_{1} \preccurlyeq T_{2}$ if and only if for each element $A \in T_{1}$ and $B \in T_{2}$, we have $H(A) \leq$ $H(B)$.

The aim of this paper is to compute the first 10 maximum value of harmonic index. We encourage the reader to consult [3-8] for basic computational techniques on the problem. 


\section{MAIN RESULTS}

In this section, we are analyzing chemical trees with $\mathrm{k}^{\text {th }}, 1 \leq \mathrm{k} \leq 10$, maximum values for the harmonic index. In order to formulate our results, we need introduce some graph notations used in this paper. Define:

$$
T_{1, H}(n)=\{M \in C T(n) \mid \forall T \in C T(n) ; H(T) \leq H(M)\},
$$

and for each $i, 1 \leq i \leq r=|C T(n)|$, we have:

$$
\left.T_{i, H}(n)=\left\{M \in C T(n)-T_{l} \cup \ldots \cup T_{i-1}\right\} \mid \forall T \in C T(n)-T_{1} \cup \ldots \cup T_{i-1} ; H(T) \leq H(M)\right\},
$$

where $r$ is the number of $n$-vertex chemical trees. The elements of $T_{i, H}(n)$ are called $i^{t h}$ maximum class of chemical trees with respect to $H$ index.

Lemma 1. Let $T_{n}$ be an $n$-vertex chemical tree and $T_{n}{ }^{\prime}$ is an $n$-vertex chemical tree obtained from $T_{n}$ by deleting a pendant vertex and appending a pendant vertex to another pendant vertex of $T_{n}$. Then $\mathrm{I}\left(T_{n}{ }^{\prime}\right) \leq \mathrm{I}\left(T_{n}\right)$.

Proof. Suppose $u v$ is a pendant edge of $T_{n}, \operatorname{deg}(v)=1, \operatorname{deg}(u) \geq 2$ and $w$ is a pendant vertex of $T_{n}$ such that $T_{n}{ }^{\prime}$ is obtained by deleting $u v$ and appending it to $w$. Then $\operatorname{deg}_{\mathrm{T}_{\mathrm{n}}}(\mathrm{w})=1=\operatorname{deg}_{\mathrm{T}_{\mathrm{n}}^{\prime}}(\mathrm{w})-1, \operatorname{deg}_{T_{n}}(u)=\operatorname{deg}_{T_{n}{ }^{\prime}}(u)+1$ and for another vertex $x$ different from $u$ and $w, \operatorname{deg}_{\mathrm{T}_{\mathrm{n}}}(\mathrm{x})=\operatorname{deg}_{\mathrm{T}_{\mathrm{n}}^{\prime}}(\mathrm{x})$. On the other hand, $I\left(T_{n}\right)=\sum_{x \neq u, w} \frac{1}{d_{x}}+\frac{1}{d_{u}}+\frac{1}{d_{w}}=$ $\sum_{x \neq u, w} \frac{1}{d_{x}}+\frac{1}{d_{u}}+1 \quad$ and $\quad \mathrm{I}\left(\mathrm{T}_{\mathrm{n}}{ }^{\prime}\right)=\sum_{\mathrm{x} \neq \mathrm{u}, \mathrm{w}} \frac{1}{\mathrm{~d}_{\mathrm{x}}}+\frac{1}{\mathrm{~d}_{\mathrm{u}}-1}+\frac{1}{\mathrm{~d}_{\mathrm{w}}+1}=$ $\sum_{x \neq u, w} \frac{1}{d_{x}}+\frac{1}{d_{u}-1}+\frac{1}{2}$. Thus $\frac{1}{d_{u}-1}+\frac{1}{2} \leq \frac{1}{d_{u}}+1$ which is equivalent to $\frac{1}{d_{u}\left(d_{u}-1\right)} \leq \frac{1}{2}$, proving the lemma.

Corollary 2. Let $T$ be an $n$-vertex chemical tree then $I(T) \geq 1+n / 2$ with equality if and only if $T$ is a path of length $n$. The second-minimum value of $I$ index is $4 / 3+n / 2$ and it is attained if and only if $T$ is isomorphic to $P_{n}^{*}$, where $P_{n}^{*}$ is a tree with exactly three pendant vertices.

Lemma 3. Let $T_{n}$ be an n-vertex chemical tree containing vertices $u$, $z$ such that $\operatorname{deg}_{T}(u)=2$ and $\operatorname{deg}_{T}(z)=2$ or 3 . Suppose $T_{1}$ and $T_{2}$ are maximal subtrees of $T_{n}$ containing $u$ as a pendant vertex and $z \in V\left(T_{2}\right)$, Figure 1 . If $T_{n}^{*}$ is the chemical tree constructed from $T_{1}$ and $T_{2}$ by identifying $u$ and $z$ then $\mathrm{I}\left(T_{n}\right) \leq \mathrm{I}\left(T_{n}^{*}\right)$, Figure 2 .

Proof. It is easy to see that $\operatorname{deg}_{\mathrm{T}_{\mathrm{n}}}(\mathrm{u})=2, \operatorname{deg}_{\mathrm{T}_{\mathrm{n}}^{*}}(\mathrm{u})=1, \operatorname{deg}_{\mathrm{T}_{\mathrm{n}}^{*}}(\mathrm{z})=\operatorname{deg}_{\mathrm{T}_{\mathrm{n}}}(\mathrm{z})+1$ and for another arbitrary vertex $\mathrm{x}, \operatorname{deg}_{\mathrm{T}_{\mathrm{n}}}(\mathrm{x})=\operatorname{deg}_{\mathrm{T}_{\mathrm{n}}^{*}}(\mathrm{x})$.

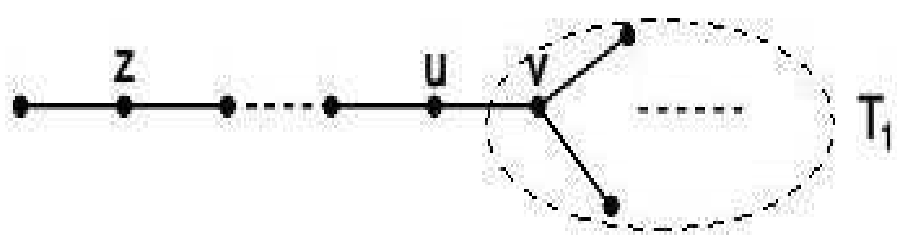

Figure 1. The Chemical Tree $T_{n}$ Containing a Subtree $T_{1}$. 


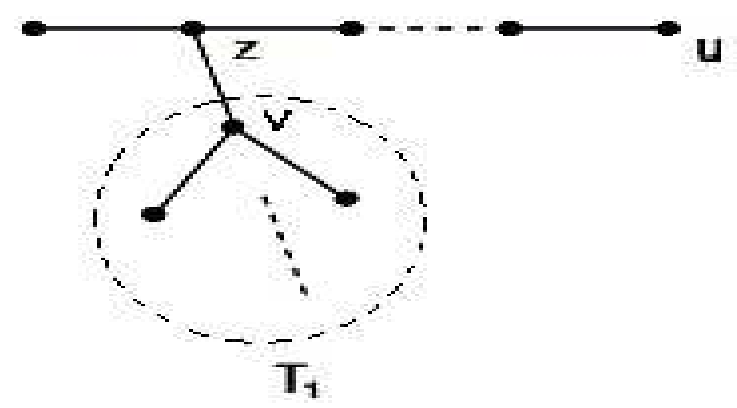

Figure 2. The Chemical Tree $T_{n}^{*}$ Constructed from $T_{1}$ and $T_{2}$.

Therefore, $\quad I\left(T_{n}\right)=\quad \sum_{x \neq z, u} \frac{1}{d_{x}}+\frac{1}{d_{u}}+\frac{1}{d_{z}} \quad=\sum_{x \neq z, u} \frac{1}{d_{x}}+\frac{1}{2}+\frac{1}{d_{z}} \quad$ and $I\left(T_{n}^{*}\right)=\sum_{x \neq z, u} \frac{1}{d_{x}}+\frac{1}{1}+\frac{1}{d_{z}+1}$. Since $\quad \frac{1}{2}+\frac{1}{d_{z}}<\frac{1}{1}+\frac{1}{d_{z}+1}, \quad \frac{1}{d_{z}}-\frac{1}{d_{z}+1}<\frac{1}{2} \quad$ and so $\frac{1}{d_{z}\left(d_{z}+1\right)}<\frac{1}{2}$, proving the lemma.

Lemma 4. Let $T_{n}$ be an n-vertex chemical tree containing vertices $u$ and $z$ of degree 3 . We also assume that $T_{1}, T_{2}$ and $T_{3}$ are maximal subtrees of $T_{n}$ with $u$ as a pendant and $z \in T_{3}$. If $T_{n}^{\prime \prime}$ is the chemical tree constructed from $T_{1}$ and $T_{2}$ by identifying $u$ and $z$ then $\mathrm{I}\left(T_{n}\right) \leq \mathrm{I}\left(T_{n}^{\prime \prime}\right)$.

Proof. By our assumption $\operatorname{deg}_{\mathrm{T}_{\mathrm{n}}}(\mathrm{u})=3, \operatorname{deg}_{\mathrm{T}_{\mathrm{n}}^{\prime \prime}}(\mathrm{u})=2, \operatorname{deg}_{\mathrm{T}_{\mathrm{n}}}(\mathrm{z})=3$ and $\operatorname{deg}_{\mathrm{T}_{\mathrm{n}}^{\prime \prime}}(\mathrm{z})=4$. Thus,

$$
\begin{aligned}
& I\left(T_{n}\right)=\sum_{x \neq z, u} \frac{1}{d_{x}}+\frac{1}{d_{u}}+\frac{1}{d_{z}}=\sum_{x \neq z, u} \frac{1}{d_{x}}+\frac{1}{3}+\frac{1}{3}, \\
& I\left(T_{n}^{\prime \prime}\right)=\sum_{x \neq z, u} \frac{1}{d_{x}}+\frac{1}{2}+\frac{1}{4},
\end{aligned}
$$

which implies that $\mathrm{I}\left(T_{n}\right) \leq \mathrm{I}\left(T_{n}^{*}\right)$.

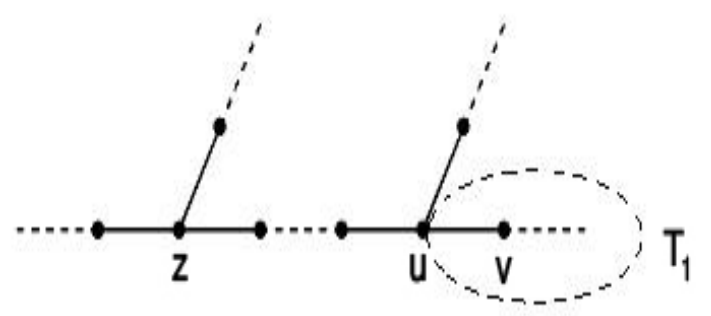

Figure 3. The Chemical Tree $T_{n}$.

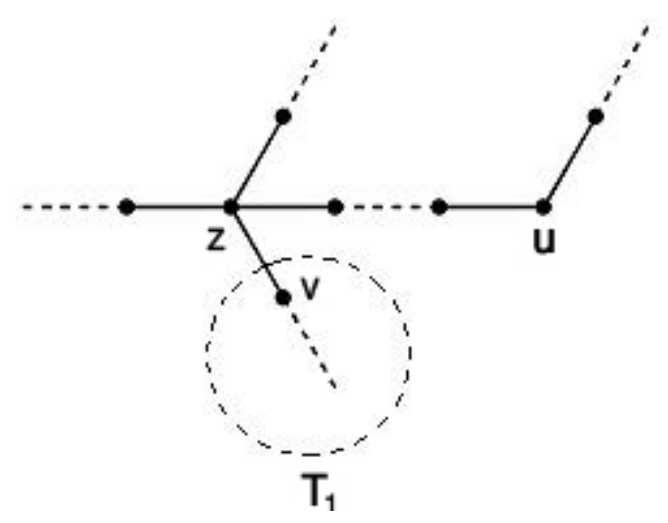

Figure 4. The Chemical Tree $T_{n}{ }^{\prime \prime}$.

Remark 5. Suppose $T$ is a chemical tree with maximum $I$ index and $r(i), i \leq 4$, denotes the number of vertices of degree $i$. Then by Lemmas 3 and $4, r(2)+r(3)=0,1$. 
In what follows $g(n)$ denotes the set of all $n$-vertex chemical trees.

Corollary 6. An $n$-vertex chemical tree $T$ has the maximum value of $I$ index in $g(n)$, if $T$ has the maximum number of pendants in $g(n)$ and non-pendant vertices satisfying $r(2)+r(3) \leq 1$.

In Table 1, the number of pendants, the number of vertices of degree 2 , the number of vertices of degree 3 and the number of vertices of degree 4 are computed for the maximal chemical trees with respect to $I$ index, when the number of vertices are at most 16 . In Table 2 , the $n$-vertex chemical trees with respect to $I$ index, $4 \leq \mathrm{n} \leq 13$, are depicted.

Table 1. The Number of Vertices of Each Degree in $n$-Vertex Chemical Trees, $4 \leq \mathrm{n} \leq 16$.

\begin{tabular}{|c|c|c|c|c|}
\hline $\begin{array}{c}\text { \# Vertices } \\
\text { of Degree 2 }\end{array}$ & $\begin{array}{c}\text { \# Vertices } \\
\text { of Degree 3 }\end{array}$ & $\begin{array}{c}\text { \# Vertices of } \\
\text { Degree 4 }\end{array}$ & $\begin{array}{c}\text { \# } \\
\text { Pendants }\end{array}$ & N \\
\hline 0 & 1 & 0 & 3 & $4=3 \times 1+1$ \\
\hline 0 & 0 & 1 & 4 & $5=3 \times 1+2$ \\
\hline 1 & 0 & 1 & 4 & $6=3 \times 1+3$ \\
\hline 0 & 1 & 1 & 5 & $7=3 \times 2+1$ \\
\hline 0 & 0 & 2 & 6 & $8=3 \times 2+2$ \\
\hline 1 & 0 & 2 & 6 & $9=3 \times 2+3$ \\
\hline 0 & 1 & 2 & 7 & $10=3 \times 3+1$ \\
\hline 0 & 0 & 3 & 8 & $11=3 \times 3+2$ \\
\hline 1 & 0 & 3 & 8 & $11=3 \times 3+3$ \\
\hline 0 & 1 & 3 & 9 & $13=3 \times 4+1$ \\
\hline 0 & 0 & 4 & 10 & $14=3 \times 4+2$ \\
\hline 1 & 0 & 4 & 10 & $15=3 \times 4+3$ \\
\hline 0 & 1 & 4 & 11 & $16=3 \times 5+1$ \\
\hline
\end{tabular}

Table 2. The Maximal Chemical Trees with respect to $I$ Index, $4 \leq \mathrm{n} \leq 13$.

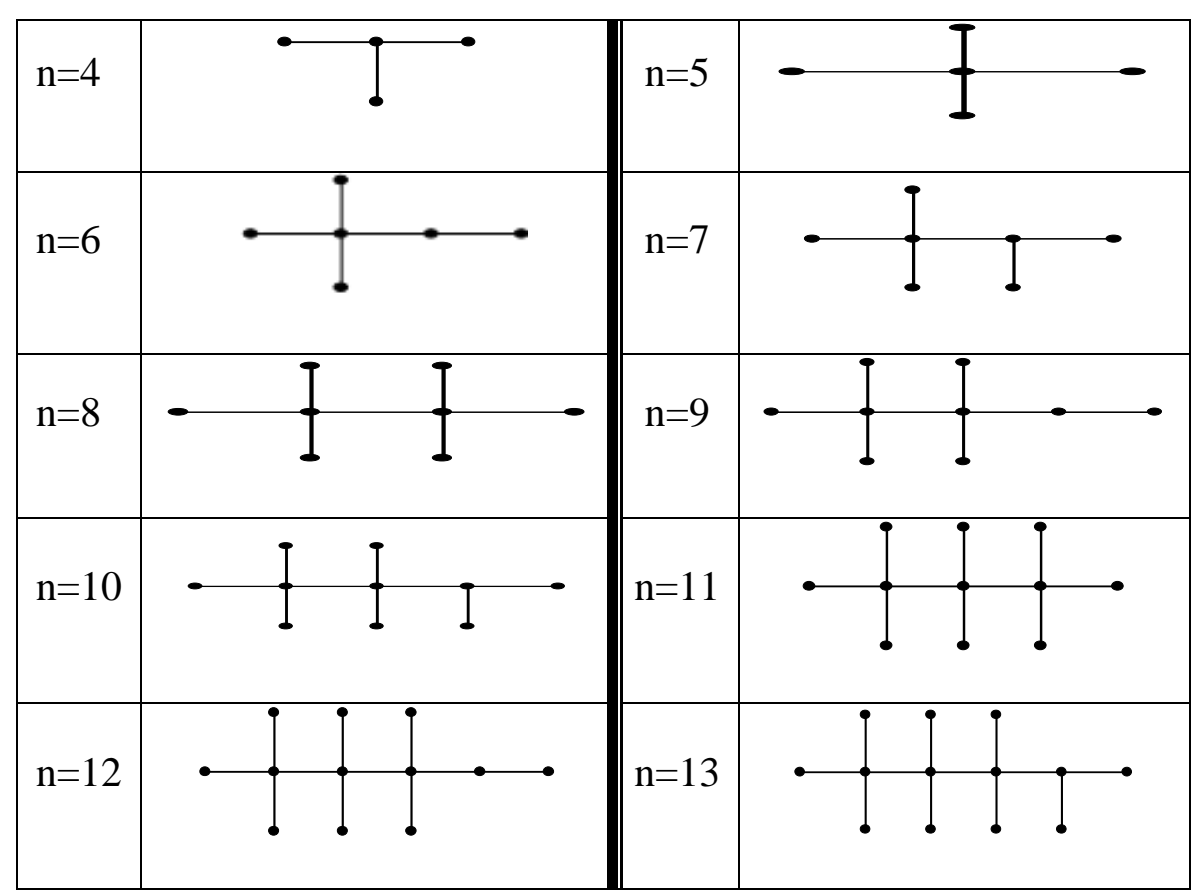


From Table 2, it is natural to ask whether or not the maximal tree with respect to $I$ index is unique. In the following example we respond negatively to this question.

Example 7. Consider the graphs $A$ and $B$ depicted in Figures 5 and 6, respectively. By simple calculations, one can see that $A$ and $B$ are non-isomorphic graphs with the same $I$ index. This shows that the maximum of I index can be occurred in more than 2 chemical trees.

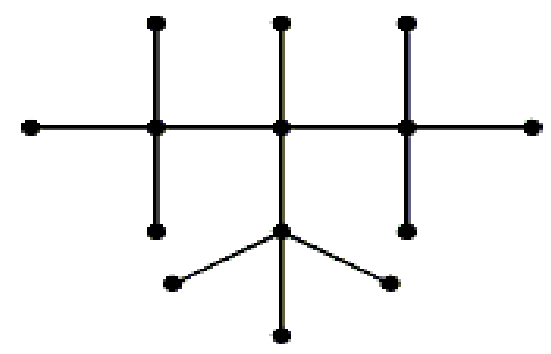

Figure 5. The Graph $A$.

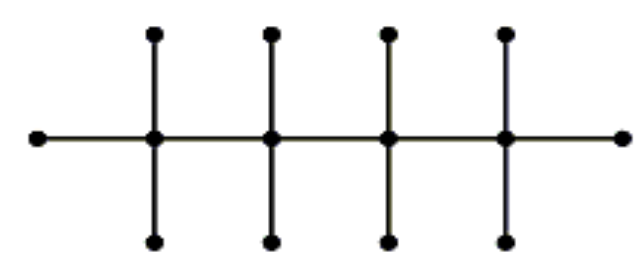

Figure 6. The Graph $B$.

Suppose $g(n)$ denotes the set of all $n$-vertex chemical trees, $n=3 k+t, 1 \leq t \leq 3, n \geq 3$ and $T \in g(n)$ is an $n$-vertex chemical tree having maximum $I$ index. One can easily seen that $r(1)=2 k+2-\lfloor 1 / t\rfloor$ and $r(2)+r(3)=1$. If $t=3$ then $r(2)=1, r(3)=0$, and if $t=1$ then $r(2)=$ 0 and $r(3)=1$. Therefore, $\mathrm{r}(4)=\mathrm{n}-\mathrm{r}(1)-\mathrm{r}(2)-\mathrm{r}(3)=\mathrm{n}-(2 \mathrm{k}+2-\lfloor 1 / t\rfloor)-(\mathrm{r}(2)+\mathrm{r}(3))=3 \mathrm{k}$ $+\mathrm{r}-2 \mathrm{k}-2+\lfloor 1 / t\rfloor-1=\mathrm{k}+\mathrm{t}-3+\lfloor 1 / t\rfloor$.

Remark 8. Suppose $T \in \mathcal{g}(n), n=3 k+t, 1 \leq t \leq 3$, and $n \geq 3$. Then the maximum of $I$ index is occurred if $r(1)=2 k+2-\lfloor 1 / t\rfloor, r(2)+r(3)=1$ and $r(4)=k+t-3+\lfloor 1 / t\rfloor$. In this case, the value of $I$ index is computed by $I(T)=2 k+2-\lfloor 1 / t\rfloor+1 / 4(k+r-3+\lfloor 1 / r\rfloor)+r(2) / 2+r(3) / 3$. Moreover, if $n=3 k+3$ then $r(2)=1, r(3)=0$ and if $n=3 k+1$ then $r(2)=0$ and $r(3)=1$.

Corollary 9. Suppose $T$ is an $n$-vertex chemical tree having maximal $I$ index in $g(n), n \geq 4$. Construct the chemical tree $S$ from $T$ by deleting a pendant connected to vertex $v$ of degree 4 in $\mathrm{T}$ and connecting to a vertex of degree 2 . If there is not a vertex of degree 2 then we connect it to a pendant. Then the chemical tree $S$ attain the second maximum value of I index.

Example 10. In this example two chemical trees $C$ and $D$ are constructed such that the number of pendants of $C$ is greater than $D$, but $I(D)<I(C)$. These are depicted in Figures 7 and 8 . It is easy to calculate $I(D)=17+1 / 4<17+1 / 6=I(C)$.

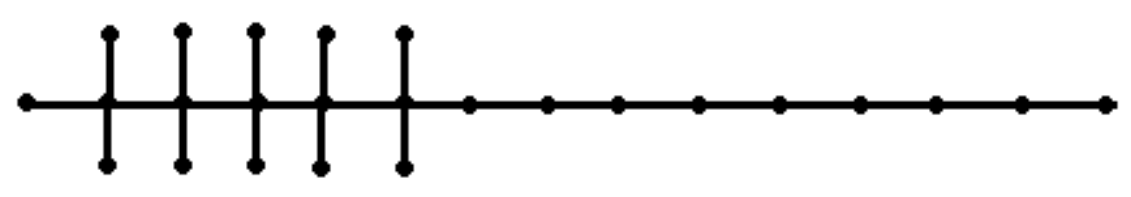

Figure 7. The Graph C.

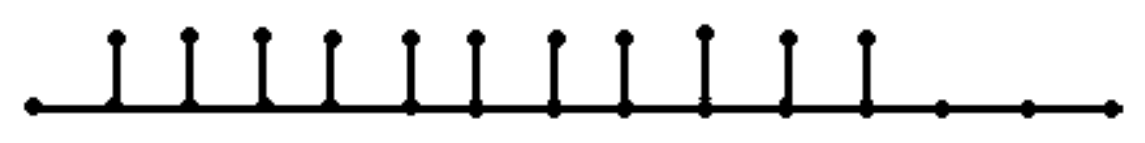

Figure 8. The Graph D. 
We now compute the first ten values of harmonic index in the class of all chemical trees. At first, it is an easy fact that the path $P_{n}$ and the chemical tree $P_{n}^{*}$ have the maximum and second maximum harmonic index, respectively.

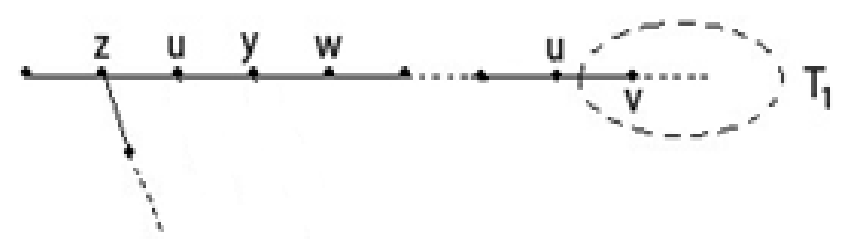

Figure 9. The Graph $P_{n}^{*}$.

To find the third maximum value of harmonic index, we consider two classes $X_{n}$ and $Y_{n}$ of chemical trees with exactly four pendant vertices in which $X_{n}$ has exactly two vertices of degree 3 and remaining vertices of degree 2 , and $Y_{n}$ has exactly one vertex of degree 4 and remaining vertices has degree 2 .

Remark 11. The third and fourth maximum values of harmonic index in the class of chemical trees will attain in $X_{n}$ and $Y_{n}$, respectively.

We are now ready to compute the fifth and sixth values of harmonic index in the class of chemical trees. We consider $n-v e r t e x$ chemical trees with five pendant vertices. These are in the form of graphs which are depicted in Figures 10 or 11. These values for the fifth and sixth maximum values of $n-$ vertex chemical trees, $n \geq 8$, are $2 n /(n+4)$ and $12 n /(6 n+25)$, respectively.

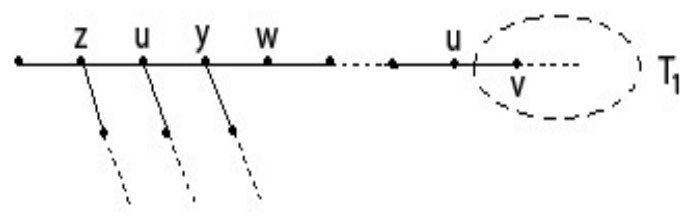

Figure 10.

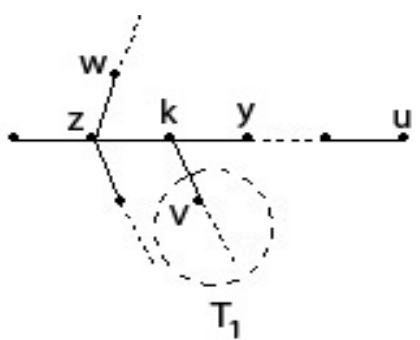

Figure 11.

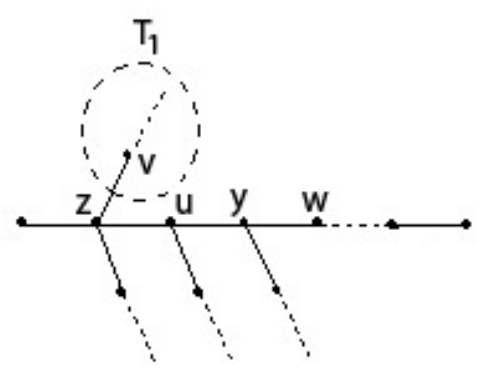

Figure 12.

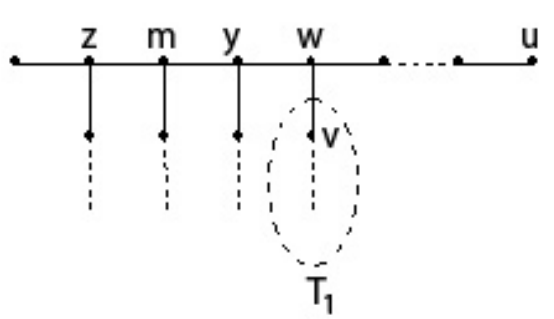

Figure 13. 


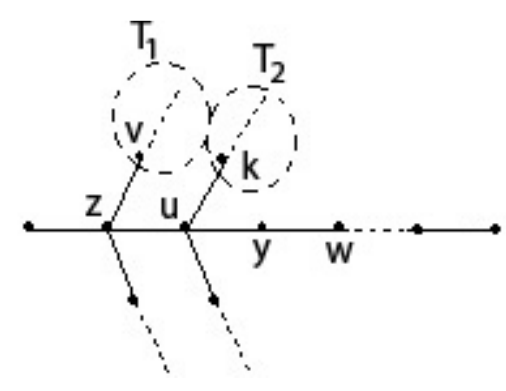

Figure 14.

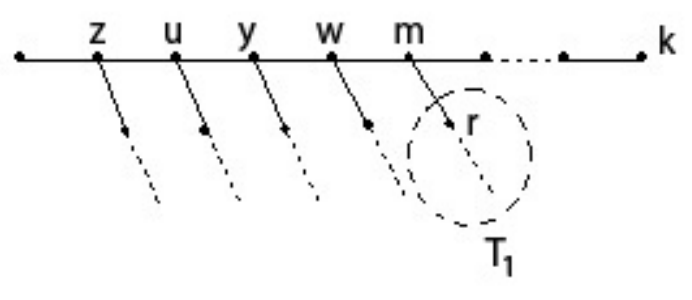

Figure 15.

Apply again our algorithm to compute the seventh, eighth and ninth maximum values in the class of all $n$-vertex chemical trees. These chemical trees have exactly six pendants and can be drawn as Figures 12-14. The tenth maximum value can be occurred in chemical trees having seven pendants. This chemical tree is depicted in Figure 15.

We record our main result in the following theorem:

Theorem 12. For each $\delta, 1 \leq \delta \leq 10, \mathrm{~T}_{\delta, \mathrm{H}}(\mathrm{n})=\left\{\mathrm{A}_{\delta, \mathrm{n}}\right\}$, where chemical trees $A_{\delta, n}$ are depicted in Table 3.

\section{Acknowledgement}

The research of the first and second authors are partially supported by the University of Kashan under grant no 159020/84.

\section{References:}

[1] D. B. West, Introduction to Graph Theory, Prentice Hall, Inc., Upper Saddle River, NJ, 1996.

[2] H. NARUMI, New topological indices for finite and infinite systems, MATCH Commun. Math. Comput. Chem. 22 (1987) 195-207.

[3] I. Gutman and M. Ghorbani, Some properties of the Narumi-Katayama index, Appl. Math. Lett. 25 (2012) 1435-1438.

[4] I. Gutman and H. Narumi, An inequality for the simple topological index, Coll. Sci. Pap. Fac. Sci. Kragujevac 11 (1990) 19-22.

[5] I. Gutman, A property of the simple topological index, MATCH Commun. Math. Comput. Chem. 25 (1990) 131-140.

[6] M. Fischermann, I. Gutman, A. Hoffmann, D. Rautenbach, D. Vidović and L. VolKmann, Extremal Chemical Trees, Z. Naturforsch. 57 a (2002) 49-52.

[7] M. J. NAdjafi-Arani, G. H. FAth-TABAR and A. R. Ashrafi, Extremal graphs with respect to the vertex PI index, Appl. Math. Lett., 22 (2009) 1838-1840.

[8] A. Zolfi and A. R. Ashrafi, Extremal properties of Narumi-Katayama index of chemical trees, Kragujevac J. Sci. 35 (2013) 71-76. 
Table 3. The $\mathrm{k}^{\text {th }}$ Maximum of Harmonic Index of Chemical Trees, $3 \leq \mathrm{k} \leq 10$.

\begin{tabular}{|c|c|c|c|c|}
\hline Names & $\mathbf{K}$ & \#Vertices & H Index & $\begin{array}{c}\text { The } \mathrm{k}^{\text {th }} \text { Maximum of Harmonic } \\
\text { Index }\end{array}$ \\
\hline $\mathrm{A}_{3,5}$ & $\mathrm{k}=3$ & $\mathrm{n}=5$ & $20 / 17$ & - \\
\hline$A_{3, n}$ & $\mathrm{k}=3$ & $\mathrm{n} \geq 6$ & $6 n /(3 n+10)$ & \\
\hline $\mathrm{A}_{4, \mathrm{n}}$ & $\mathrm{k}=4$ & $\mathrm{n} \geq 6$ & $4 n /(2 n+7)$ & \\
\hline $\mathrm{A}_{5,7}$ & $\mathrm{k}=5$ & $\mathrm{n}=7$ & $84 / 67$ & \\
\hline $\mathrm{A}_{5, \mathrm{n}}$ & $\mathrm{k}=5$ & $\mathrm{n} \geq 8$ & $2 n /(n+4)$ & \\
\hline $\mathrm{A}_{6, \mathrm{n}}$ & $\mathrm{k}=6$ & $\mathrm{n} \geq 8$ & $12 n /(6 n+25)$ & \\
\hline $\mathrm{A}_{7,8}$ & $\mathrm{k}=7$ & $\mathrm{n}=8$ & $16 / 13$ & - \\
\hline $\mathrm{A}_{7,9}$ & $\mathrm{k}=7$ & $\mathrm{n}=9$ & $108 / 83$ & • \\
\hline $\mathrm{A}_{7, \mathrm{n}}$ & $\mathrm{k}=7$ & $\mathrm{n} \geq 10$ & $6 n /(3 n+14)$ & \\
\hline $\mathrm{A}_{8,9}$ & $\mathrm{k}=8$ & $\mathrm{n}=9$ & $9 / 7$ & \\
\hline $\mathrm{A}_{8, \mathrm{n}}$ & $\mathrm{k}=8$ & $\mathrm{n} \geq 10$ & $12 n /(6 n+29)$ & \\
\hline$A_{9, n}$ & $\mathrm{k}=9$ & $\mathrm{n} \geq 10$ & $2 n /(n+5)$ & \\
\hline $\mathrm{A}_{10,10}$ & $\mathrm{k}=10$ & $\mathrm{n}=10$ & $60 / 47$ & \\
\hline $\mathrm{A}_{10,11}$ & $\mathrm{k}=10$ & $\mathrm{n}=11$ & $4 / 3$ & \\
\hline $\mathrm{A}_{10, \mathrm{n}}$ & $k=10$ & $\mathrm{n} \geq 12$ & $6 n /(3 n+16)$ & \\
\hline
\end{tabular}

